\title{
Efficacy of wrist robot-aided orthopedic rehabilitation: a randomized controlled trial
}

\author{
Giulia Aurora Albanese ${ }^{1,2^{*}}$, Elisa Taglione ${ }^{3}$, Cecilia Gasparini ${ }^{3}$, Sara Grandi ${ }^{3}$, Foebe Pettinelli ${ }^{3}$, Claudio Sardelli ${ }^{3}$, \\ Paolo Catitti ${ }^{3}$, Giulio Sandini ${ }^{1}$, Lorenzo Masia ${ }^{4}$ and Jacopo Zenzeri ${ }^{1}$
}

\begin{abstract}
Background: In recent years, many studies focused on the use of robotic devices for both the assessment and the neuro-motor reeducation of upper limb in subjects after stroke, spinal cord injuries or affected by neurological disorders. Contrarily, it is still hard to find examples of robot-aided assessment and rehabilitation after traumatic injuries in the orthopedic field. However, those benefits related to the use of robotic devices are expected also in orthopedic functional reeducation.

Methods: After a wrist injury occurred at their workplace, wrist functionality of twenty-three subjects was evaluated through a robot-based assessment and clinical measures (Patient Rated Wrist Evaluation, Jebsen-Taylor and Jamar Test), before and after a 3-week long rehabilitative treatment. Subjects were randomized in two groups: while the control group $(n=13)$ underwent a traditional rehabilitative protocol, the experimental group $(n=10)$ was treated replacing traditional exercises with robot-aided ones.
\end{abstract}

Results: Functionality, assessed through the function subscale of PRWE scale, improved in both groups (experimental $p=0.016$; control $p<0.001)$ and was comparable between groups, both pre $(U=45.5, p=0.355)$ and post $(U=47$, $p=0.597)$ treatment. Additionally, even though groups' performance during the robotic assessment was comparable before the treatment $(U=36, p=0.077)$, after rehabilitation the experimental group presented better results than the control one $(U=26, p=0.015)$.

Conclusions: This work can be considered a starting point for introducing the use of robotic devices in the orthopedic field. The robot-aided rehabilitative treatment was effective and comparable to the traditional one. Preserving efficacy and safety conditions, a systematic use of these devices could lead to decrease human therapists' effort, increase repeatability and accuracy of assessments, and promote subject's engagement and voluntary participation. Trial Registration ClinicalTrial.gov ID: NCT04739644. Registered on February 4, 2021 -Retrospectively registered, https:// www.clinicaltrials.gov/ct2/show/study/NCT04739644.

Keywords: Robotic rehabilitation, Robotic assessment, Wrist injury, Orthopedic

\section{Background}

Wrist traumatic injuries usually lead to hand motor control deficits and loss of functionality, as direct consequences of both the lesion itself and the following

\footnotetext{
*Correspondence: giulia.albanese@iit.it

${ }^{1}$ Robotics, Brain and Cognitive Sciences (RBCS) Unit, Istituto Italiano di Tecnologia, Genoa, Italy

Full list of author information is available at the end of the article
}

immobilization period. Actually, occurrence of tissue rigidity, lack of stretch, muscle strength reduction, pain and edema could bring to a limited Range Of Motion (ROM) along some directions of movement [1]. Moreover, long periods of immobilization could take to proprioceptive deficits, preventing post-traumatic subjects to have a proper control of movements and worsening their performance during fine manipulation tasks [2-4]. Wrist injuries could differ in terms of severity and site: 
lesions may involve flexor or extensor tendons, complexes made of fibrocartilage, ligaments, bones or more than one of these tissues [1]. Each tissue presents a different vascularization and consequently a different healing time [5], while the site of the lesion has a direct influence on the resulting functional impairment, such as the movement direction more compromised [6]. For these reasons and because of age differences, both orthopedic treatments and following rehabilitative approaches could differ between subjects [7-10]. In terms of orthopedic treatments, we can identify conservative and surgery interventions: while the former implies the application of a cast or a splint, the latter allows to shorter periods of immobilization, decreasing all the related problems, such as rigidity, loss of strength and altered proprioception [11]. Generally, the choice of a surgical or non-surgical approach seems to have an influence on the grip force, reduced for non-surgical cases, but it has been demonstrated the absence of significant differences in terms of motor and sensory impairments [3]. Removed the cast or splint, rehabilitation programs should begin as soon as possible. Rehabilitative interventions have the goal of restoring functional abilities and subject's self-sufficiency: therapists tailor these protocols to meet the need presented by individual patients, in terms of duration, intensity and exercises. A large variety of exercises is conventionally used in clinics: active and passive joint mobilizations, continuous motion and strengthening exercises supervised and assisted by physical therapists, supportive splints, physical methods of pain management, but also occupational therapy programs and self-administered exercises [7-10]. However, some standardized protocols of ordinary physical interventions are usually applied [10]: treated the presence of pain and edema, initially only active exercises to stretch soft tissues and improve the range of motion are allowed; passive mobilizations, strengthening and proprioceptive exercises are gradually introduced in the following weeks [7, 8]. Particularly, both active and passive exercises for increasing the ROM have a relevant level of evidence supporting their usage [12].

Nowadays, physical therapists work with traditional devices for therapeutic exercise, common in every rehabilitative center, or employ their own strength for manual mobilizations. In contrast to what has been happening in the neuro-rehabilitative field [13-15], in the orthopedic one examples of robot-aided assessment and rehabilitation of wrist injuries are hard to find [16, 17]. However, analogously to what has been observed in the neuromotor reeducation of the upper limb in subjects after stroke or spinal cord injuries [18-21], advantages are expected from the use of these devices for the functional reeducation after wrist traumatic injuries. Robot-assisted therapy meets the need of orthopedic patients for a personalized protocol and a maximized training effect, allowing levels of assistance or resistance tailored on the real-time performance. Actually, high-resolution recording of spatial and temporal data allows to compute novel performance indicators $[6,22,23]$ and document constantly progresses related to therapy, assessed under repeatable and safe conditions. For these reasons, the use of a robotic system with post-traumatic subjects could decrease human therapists' effort and increase the efficiency in terms of both treatment duration and final reached functionality. Finally, the possibility to couple therapy with a virtual reality environment could be useful to increase patient's participation, engagement and motivation, demonstrated to be related to the treatment success or failure [17, 24-26].

For all the above-mentioned reasons, this randomized clinical trial aimed to address three main questions about the use of a robotic device for the rehabilitative training of subjects presenting wrist injuries: (1) whether a robot-based rehabilitative approach is effective on wrist functionality; (2) whether the effects of robot-based rehabilitation are different from those achieved through a conventional therapy; (3) which is the acceptability of this novel approach perceived by patients. In the present work, we introduce the structure of the clinical trial and the results obtained from robotic evaluations, clinical measures and asked approval rating.

\section{Methods \\ Experimental setup}

The study was carried out at the INAIL Motor Rehabilitation Center (Volterra, Italy) and involved the employment of the WRISTBOT, a robotic device developed at the Istituto Italiano di Tecnologia (Genoa, Italy) [27, 28]. This robot was designed for and is currently employed in motor control and rehabilitation studies of the human wrist $[23,29-31]$. The WRISTBOT is a fully backdrivable manipulandum that allows for movements along its 3 Degrees of Freedom (DoFs) in a human-like Range Of Motion (ROM) of the wrist: $\pm 62^{\circ}$ flexion/extension (FE), $-40^{\circ} \%+45^{\circ}$ in radial/ulnar deviation (RUD), and $\pm 60^{\circ}$ pronation/supination (PS). In addition, the robot permits motions along planes that involve combined multi-DoFs movements. Mechanically, the robot was developed to have low values of inertia, emulating the fluency of natural movements. Each DOF is measured by high resolution incremental encoders and actuated by one brushless motor or two in case of the RUD planes, providing both gravity compensation and continuous torque values necessary to manipulate the human wrist joints. The torque ranges at the different wrist joints are $1.53 \mathrm{Nm}$ on FE, $1.63 \mathrm{Nm}$ on RUD and 2.77 Nm on PS. Depending on the 
torques exerted, the device can be used in either active or assistive/passive modality. While the active modality requires only subject's active muscle work, the assistive/passive one was implemented using an impedance control scheme, based on the real-time relative position between the target and the end-effector, with a $1 \mathrm{kHz}$ sampling frequency. The system is integrated with a Virtual Reality environment (VR), useful to provide a visual feedback to the user while he/she is requested to complete the tasks.

\section{Subjects and experimental protocol}

The design of this study was an interventional, parallel, and randomized clinical trial on a consecutive convenience sample of 27 subjects. Using a computer, a therapist randomly assigned subjects to each group, whose main characteristics are reported in Table 1. Twentythree subjects completed the entire protocol, because of three dropouts in the experimental group $(n=10)$ and one among control subjects $(n=13)$. The dropouts were due to the impossibility to have a constant routine of at least 4 training sessions performed each week. In particular, some inclusion criteria have to be fulfilled to participate the study: adults of both sexes, aged between 18 and 65 years, presenting functional and spatial limitations of the wrist joint, following an injury occurred at their workplace. Participants' injuries included scapholunate ligament injuries, distal radius/ ulnar fractures, carpal bones fractures or dislocations, triangular fibrocartilage complex (TFCC) injuries. In details, subjects had to be in the post-immobilization phase and the temporal distance from the acute event did not have to exceed 6 months. Exclusion criteria were non-compliance with study requirements, pregnancy or breast feeding, prior history of malignancy, contraindications to wrist passive movements, acute inflammatory arthritis of the wrist, open skin at the level of the patient-device interface. The research was performed in accordance with the Declaration of Helsinki and approved by the local ethics committee (protocol number 76, code CRMINAIL03). An informed consent was signed to participate to the study.

During robot-aided sessions, subjects sat on a chair in front of a screen, holding the handle of the robot, with their forearm strapped to the robot support to assure a correct alignment between the axes of the mechanical structure and the wrist's rotational ones (Fig. 1).

The protocol included both assessment and rehabilitative sessions (Fig. 2). All subjects performed the same sessions of assessment, which included two evaluations through the robotic system and clinical measures at the beginning $\left(\mathrm{T}_{\mathrm{b}}\right)$ and at the end $\left(\mathrm{T}_{\mathrm{e}}\right)$ of the rehabilitative training, and a follow up through phone call, three months after the end of the treatment $\left(T_{f}\right)$.

Three clinical measures were assessed at $\mathrm{T}_{\mathrm{b}}$ and $\mathrm{T}_{\mathrm{e}}$ as primary outcomes:

1. Jamar Test [32]: using a hand dynamometer, subjects performed three trials to evaluate the mean static palmar force exerted in $\mathrm{kg}$.

2. Jebsen Taylor Hand Function Test (JTHFT) [33, 34]: consisting of six items, its aim was to evaluate dexterity in terms of fine motor skills, weighted functional tasks and non-weighted functional tasks. Each item is scored according to the time taken to complete the task.

3. Patient Rated Wrist/Hand Evaluation (PRWE) [3537]: a questionnaire composed of a pain (PRWE-P) and a function (PRWE-F) subscale. Each subsection has a maximum score of 50 and a minimum of 0 , where less score points out a better performance. PRWE was the only assessment involved also in the follow-up call $\left(\mathrm{T}_{\mathrm{f}}\right)$.

Table 1 Subjects' characteristics and distribution in the experimental and control group

\begin{tabular}{lll}
\hline & Experimental $(\mathbf{n}=\mathbf{1 0})$ & Control $(\mathbf{n}=\mathbf{1 3})$ \\
\hline Sex (male/female) & $5 / 5$ & $9 / 4$ \\
Age (mean \pm std) & $48.7 \pm 11.8$ years & $50.9 \pm 9.1$ years \\
Right-handed & 9 & 11 \\
Dominant side injured & 6 & 8 \\
Lesion: & 5 & 8 \\
Fractures/dislocations & 3 & 5 \\
Ligament injuries & 1 & - \\
Both & 1 & - \\
Others (TFCC lesion) & $8 / 2$ & $10 / 3$ \\
Orthopedic treatment (surgery intervention/conservative treatment) & $101.60 \pm 43.3$ \\
Temporal distance between the acute event and the 1st evaluation (days, mean \pm SD) & $98.5 \pm 44.0$ & \\
\hline
\end{tabular}




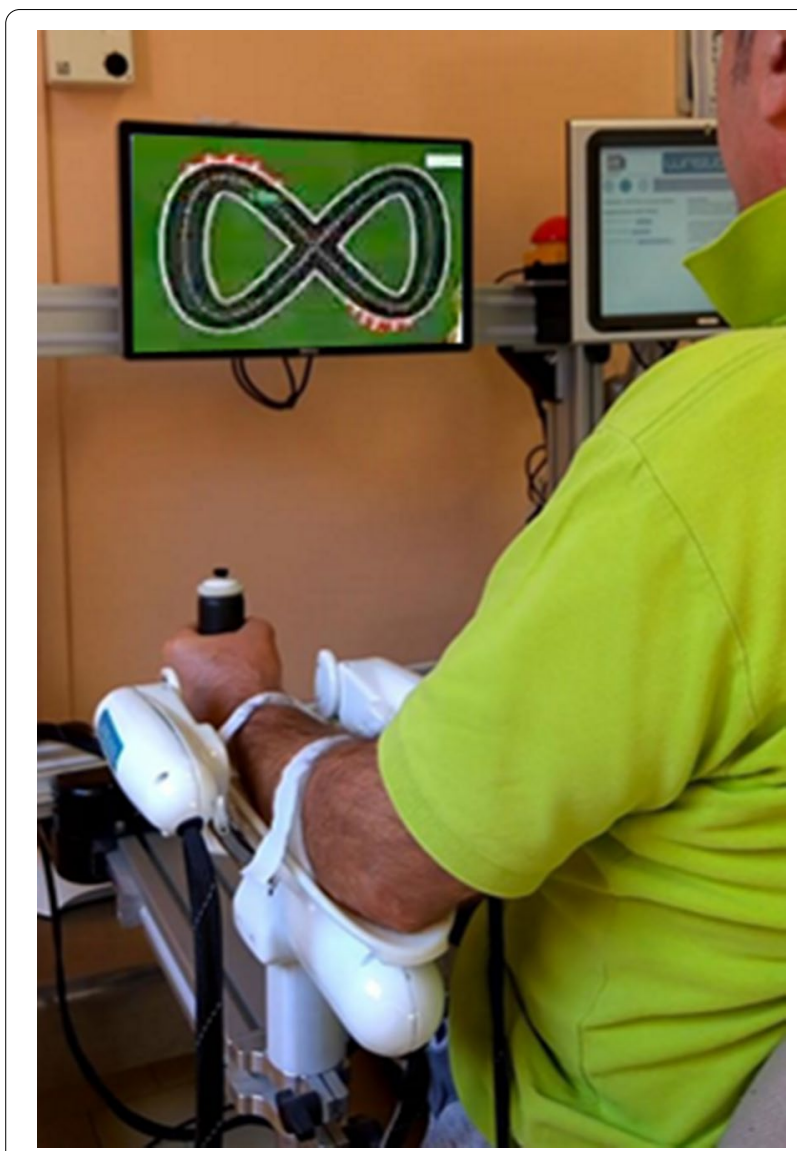

Fig. 1 The experimental setup. Subjects' placement and virtual reality during an illustrative example of a tracking task

On the same days $\left(\mathrm{T}_{\mathrm{b}}\right.$ and $\left.\mathrm{T}_{\mathrm{e}}\right)$, subjects performed also the robot-aided evaluation. Each robotic assessment lasted around $45 \mathrm{~min}$ and followed a 15 -min session to familiarize with the device, assuring all subjects to be similarly acquainted with the device. Rehabilitative training was not performed during assessment days.
Robot-aided evaluative session included five exercises, useful to assess robotic primary outcomes as ROM, exerted forces, dexterity, and wrist position sense acuity:

1. Passive ROM Starting from the neutral position $\left(0^{\circ}\right.$ along each DoF), the device moved the wrist of the subject along different directions until subject's maximum tolerance, notified by himself/herself pushing a button with the not injured hand. Subjects let the robot moving their wrist passively, without opposing or facilitating the motion. Target directions were 8 equally distributed in the FE/RUD space [radial deviation (RD), ulnar deviation (UD), flexion F, extension (E), ulnar flexion (UF), ulnar extension (UE), radial flexion (RF) and radial extension (RE)], and 2 along PS [pronation (P), supination (S)]. Passive ROM assessment was necessary to choose appropriate target positions and distances in some of the following exercises (Target Tracking and Joint Position Matching), suitable for the severity of the injury of the single subject and his/her level of healing. Outcome measures consisted in the maximum ROM in degrees achieved along each direction.

2. Active ROM From the initial neutral position, subjects moved actively the device as far as they could, along the same directions of the previous exercise. Any assistive force was applied, but the weight of the device was compensated during active motions. The outcome measure was the maximum active ROM in degrees achieved along each direction.

3. Isometric Force While the device kept subjects on the wrist neutral position, they were requested to perform a maximal contraction toward different directions. While subjects pushed towards each target direction, the device resisted to the imposed force, such that no motion was performed. The outcome measure was maximal peak force in Newton meas-

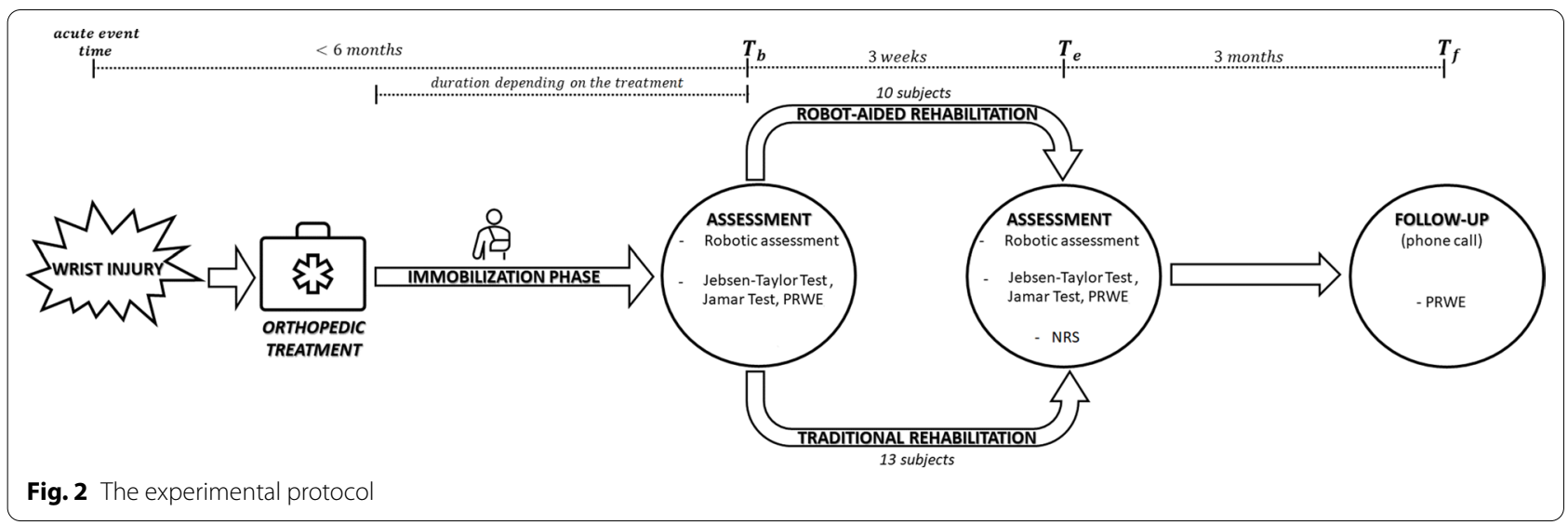


ured along each direction (same directions as in ROM assessment).

4. Target Tracking Subjects had to follow a target moving on a first order Lissajous trajectory, showed on the screen two-dimensional space. Subjects performed two laps, actively moving in two different directions of rotation (counter and clockwise) across the space described by combinations of FE and RUD motions. The size of the figure was determined by the $75 \%$ of the smallest assessed ROM among UD, RD, $\mathrm{F}$ and $\mathrm{E}$ directions. The resulting outcome measure was the mean figural error in degrees, i.e. the average angular distance between target and end-effector trajectory in each sampled point [38].

5. Joint Position Matching While the subject was blindfolded, the device moves his/her wrist in a defined direction, until the $75 \%$ of the subject's ROM along that direction. After $3 \mathrm{~s}$, the wrist was passively brought back to the neutral position. Then, maintaining the blindfolded condition, the subject was asked to reproduce the joint configuration previously assumed passively. Target directions corresponded to the same directions along which the ROM has been assessed. Performance was measured in terms of matching error, i.e., the Euclidean distance between target and matched points. Matching error was measured in degrees, since each-DoF rotational measurements were considered as single coordinates to compute distances [39].

In order to avoid inflammatory issues, each exercise was repeated once during each evaluation.

Finally, subjects were asked to indicate their approval rating through a Numerical Rating Scale (NRS) [40, 41]: subjects' level of satisfaction about treatment was asked as secondary outcome at the end of the rehabilitative treatment $\left(\mathrm{T}_{\mathrm{e}}\right)$.

Concerning the rehabilitative training, the protocol included a three-week long rehabilitation, during which subjects performed 4/5 sessions per week, since the first and the last day were exclusively dedicated to the assessment. Each session lasted around $90 \mathrm{~min}$ and its structure was individually chosen by the medical specialist, according to the severity of the injury of each single subject. The control group was treated with conventional rehabilitative treatments, supervised and assisted by a therapist, using traditional tools and devices necessary for therapeutic exercises.

The experimental group underwent a comparable rehabilitative treatment, decided and supervised by the medical specialist, except for the replacement of some traditional manual exercises with robot-based ones. In details, replaced training exercises aimed to improve the ROM, muscle strength and dexterity (Table 2).

Before each robotic training session, the passive ROM of participants was assessed through the dedicated exercise, because of safety reasons and in order to tailor target positions in the immediately following rehabilitative session. To avoid a learning effect related to a more prolonged use of the device in the experimental group, therapeutic robot-aided exercises were designed with the aim to be deeply different from those used in the sessions of assessment.

\section{Data processing}

Given each-DoF encoder recordings during robotic assessments, data of joint rotations were re-sampled at a uniform $100 \mathrm{~Hz}$ sample rate by linear interpolation and filtered with a sixth order Savitzky-Golay low-pass filter ( $8 \mathrm{~Hz}$ cut-off frequency). While these represented performed angular displacements, the amount of current delivered by motors was used to estimate the forces exerted to assist or resist to subjects' motion.

Computed the above-mentioned outcome measures for each exercise (see "Subjects and experimental protocol"), we obtained and statistically tested single-subject performance along each direction involved in the five exercises included in the assessment. Data smaller than 1.5 of the interquartile range (IQR) from the first quartiles or bigger than 1.5 IQR from the third one were considered outliers.

Additionally, we chose to inspect the whole performance of single exercises. The issue we had to face here was that, even in healthy populations, motor or perceptive performance along different directions is not comparable. For these reasons, we normalized single-direction performance of each subject respect to our sample performance along that direction. In details, for each subject and exercise, each single-direction outcome measure $x$ was normalized as shown in the following equation:

$$
x_{\text {norm }}=\frac{x_{\text {best }}-x}{x_{\text {best }}-x_{\text {worst }}}
$$

where $x_{\text {best }}$ and $x_{\text {worst }}$ represent respectively the best and worst performance found along that specific direction of the considered exercise, among all the subjects and evaluations. In particularly, while in case of ROM or force exerted a better performance is reflected in higher output values, in all the other exercises higher outcome measures represented worse performances, since in those cases our metric was an error. Since our sample presented a wide range of injuries and different functional issues, some subjects showed values comparable to not-injured subjects [22, 39], already at the first evaluation. Therefore, 


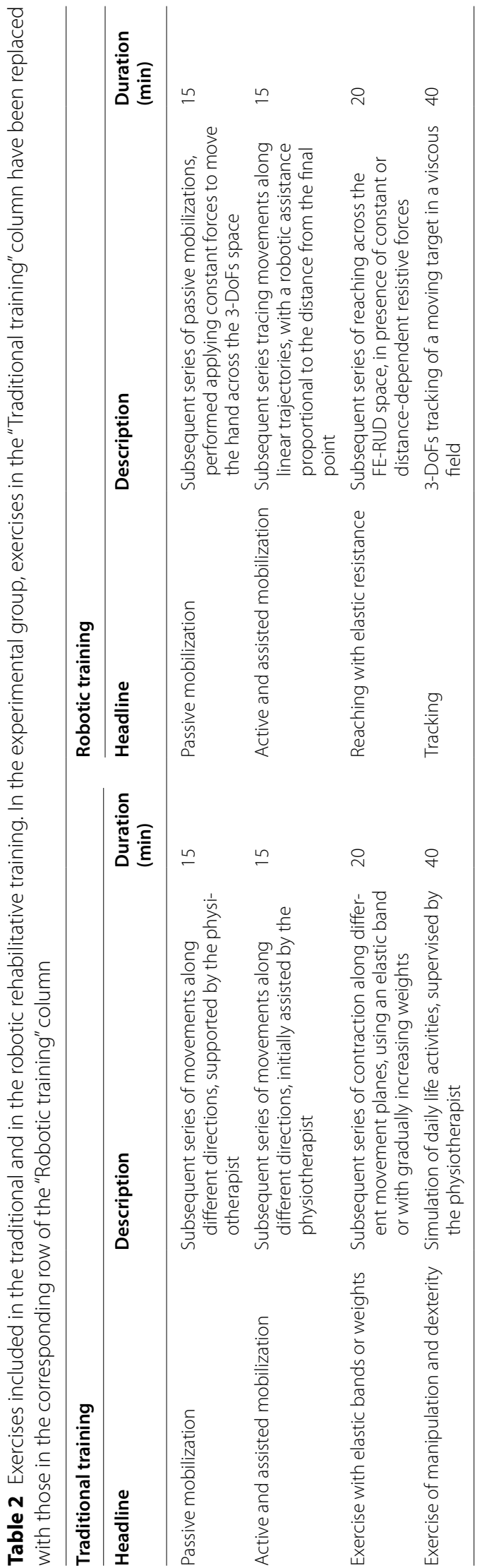


this normalization scaled our measures respect to a range from a not injured to an injured wrist performance, along each specific direction of each exercise. Computed values resulted to be in a $[0,1]$ range, where lower values represented a better performance.

Next, for each exercise, we computed the median value within normalized single-directions, getting a single outcome measure in the $[0,1]$ range for each exercise (outcome $_{\text {exercise }}$ ), whose meaning was how far from a healthy-like wrist functionality goal the subject performed.

Finally, given one outcome indicator for each out of the five exercises in each session of assessment (outcome exercise), we compute an equally-weighted linear combination of them (Eq. 2):

$$
\text { Robotic Assessment Index }=\sum_{\text {exercise }=1}^{5} \text { outcome }_{\text {exercise }}
$$

Briefly, for each subject, this score (Robotic Assessment Index) reflected the global performance during single sessions of robotic assessment, with lower values suggesting a better performance.

\section{Statistical analysis}

Statistical analysis involved single-direction measures, single-exercise outcomes, the Robotic Assessment Index, clinical measures and NRS results. Normality of data was inspected through Shapiro-Wilk Tests: these revealed presences of non-normally distributed data, which led to choose non-parametric tests for the statistical analysis. Mann-Whitney U tests have been performed to statistically analyze between-group performance at each session of assessment $\left(\mathrm{T}_{\mathrm{b}}, \mathrm{T}_{\mathrm{e}}\right.$ and $\left.\mathrm{T}_{\mathrm{f}}\right)$. Dependent non-parametric Wilcoxon Matched Pairs tests have been used to compare outcome metrics at $\mathrm{T}_{b}$ with those at $\mathrm{T}_{e}$, within each group (experimental/ control). Conversely, since PRWE was the only metric assessed three times $\left(\mathrm{T}_{\mathrm{b}}, \mathrm{T}_{\mathrm{e}}\right.$ and $T_{f}$ ), PRWE of each group was statistically analyzed through Friedman tests. Whether a main effect of measurement time was found, post-hoc pairwise comparisons were conducted through Durbin-Conover tests. Multiple comparisons were adjusted with a Bonferroni correction. Differences were considered significant when $\mathrm{p}<0.050$. Jamovi Statistical Data Analysis tool (JSDA, version 1.2.27) was used to conduct statistical analysis.

\section{Results}

\section{Clinical measures}

Figure 3 show what has been found from clinical tests conducted by therapists, on the same days during which the robotic assessments were performed. The Jamar Test, assessing the grip force, showed values at $\mathrm{T}_{\mathrm{e}}$ greater than $\mathrm{T}_{\mathrm{b}}$ : even though groups did not present significant differences both pre $(\mathrm{U}=57, \mathrm{p}=0.642)$ and post rehabilitation $(\mathrm{U}=45, \mathrm{p}=0.222)$, the grip force increase from $\mathrm{T}_{\mathrm{b}}$ to $\mathrm{T}_{\mathrm{e}}$ was significant in the control group (experimental: $\mathrm{W}=6, \mathrm{p}=0.058$; control: $\mathrm{W}=12, \mathrm{p}=0.021$ ). Conversely, in the Jebsen-Taylor test, only the experimental group presented a significant improvement (experimental: $\mathrm{W}=52, \mathrm{p}=0.010$; control: $\mathrm{W}=43, \mathrm{p}=0.126$ ), despite initial and final scores comparable between groups $\left(\mathrm{T}_{\mathrm{b}}\right.$ : $\left.\mathrm{U}=58.5, \mathrm{p}=0.710 ; \mathrm{T}_{\mathrm{e}}: \mathrm{U}=42, \mathrm{p}=0.387\right)$. Friedman tests on PRWE revealed that rehabilitation had effects mainly on the function subscale: pain subscale results presented neither significant difference between groups at all evaluations $\left(\mathrm{T}_{\mathrm{b}}: \mathrm{U}=49.5, \mathrm{p}=0.774 ; \mathrm{T}_{\mathrm{e}}: \mathrm{U}=46, \mathrm{p}=0.594\right.$; $\mathrm{T}_{\mathrm{f}}: \mathrm{U}=26, \mathrm{p}=0.372$ ), nor single-group improvements, represented by absence of significantly different scores among evaluations (experimental: $\mathrm{X}^{2}=2.82, \mathrm{p}=0.244$;

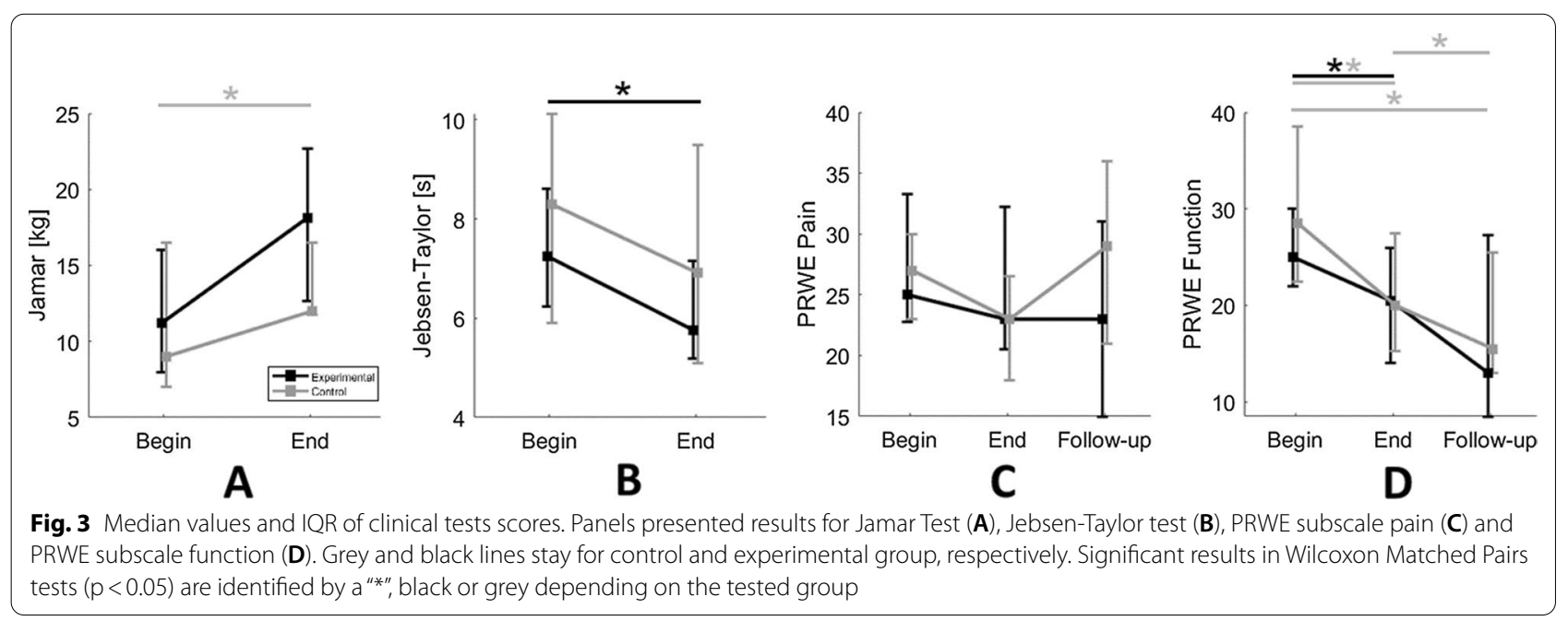


control: $x^{2}=2.17, p=0.338$ ). On the other hand, both rehabilitative treatments had a significant effect on the function subscale (experimental: $x^{2}=2.84, p=0.016$; control: $\left.x^{2}=16.8, \quad \mathrm{p}<0.001\right)$. Pairwise comparison showed that, while the control group kept on improving also after 3 months $\left(\mathrm{T}_{\mathrm{b}}\right.$ vs $\mathrm{T}_{\mathrm{e}}$ : $\mathrm{D}-\mathrm{C}=5.49, \mathrm{p}<0.001 ; \mathrm{T}_{\mathrm{b}}$ vs $\mathrm{T}_{\mathrm{f}} \mathrm{D}-\mathrm{C}=7.82, \mathrm{p}<0.001 ; \mathrm{T}_{\mathrm{e}}$ vs $\left.\mathrm{T}_{\mathrm{f}}: \mathrm{D}-\mathrm{C}=2.32, \mathrm{p}=0.012\right)$, the experimental group did not reveal any significant change from the end of the treatment to the follow-up call $\left(\mathrm{T}_{\mathrm{b}}\right.$ vs $\mathrm{T}_{\mathrm{e}}: \mathrm{D}-\mathrm{C}=2.65, \mathrm{p}=0.036 ; \mathrm{T}_{\mathrm{b}}$ vs $\mathrm{T}_{\mathrm{f}}: \mathrm{D}-\mathrm{C}=4.08$, $\mathrm{p}=0.088 ; \mathrm{T}_{\mathrm{e}}$ vs $\left._{\mathrm{f}}: \mathrm{D}-\mathrm{C}=1.43, \mathrm{p}=1.000\right)$. Nevertheless,

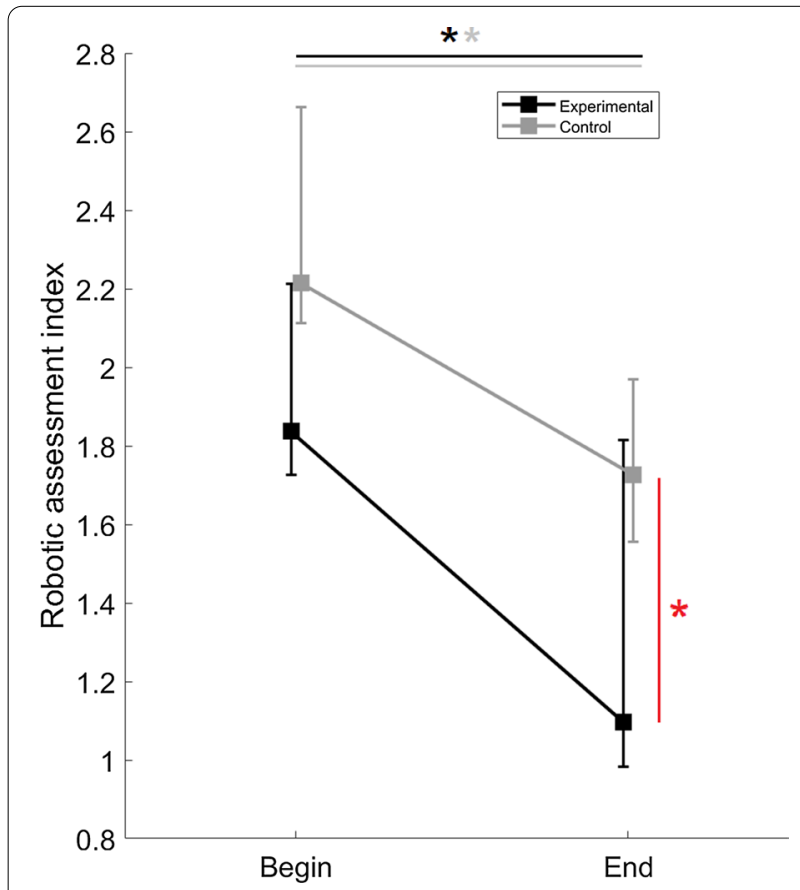

Fig. 4 Median values and IQR of Robotic Assessment Index. Grey and black lines stay for control and experimental group, respectively. Significant results in Wilcoxon Matched Pairs tests are identified by a "*", black for the experimental group and grey for the control one. Red "*" identified significant differences found after independent MannWhitney $U$ tests independent Mann-Whitney $\mathrm{U}$ tests revealed that group scores were anyhow comparable at each evaluation $\left(\mathrm{T}_{\mathrm{b}}\right.$ : $\mathrm{U}=45.5, \mathrm{p}=0.355 ; \mathrm{T}_{\mathrm{e}}: \mathrm{U}=47, \mathrm{p}=0.597 ; \mathrm{T}_{\mathrm{f}}: \mathrm{U}=37$, $\mathrm{p}=0.703)$.

\section{Robotic Assessment Index}

Figure 4 shows the scores of each group during single sessions of robotic assessment, computed considering the performance of all the five exercises. Wilcoxon Matched Pairs tests showed that both robotic and traditional training led to an improved performance in the robotaided assessment after rehabilitation (experimental: $\mathrm{W}=54, \mathrm{p}=0.004$; control: $\mathrm{W}=87, \mathrm{p}=0.002)$. However, although groups were comparable before the rehabilitative treatment $(U=36, p=0.077)$, Mann-Whitney $U$ tests revealed that the experimental group presented a significantly lower Robotic Assessment Index after the rehabilitative treatment $(U=26, p=0.015)$, pointing out a final performance better than the control group. Given this global idea, we moved to analyze single exercises of assessment, with the aim of understanding the weight each component had on the whole assessment.

\section{Range of motion}

As described by median values and IQRs in Table 3, groups presented comparable values of both active and passive $\mathrm{ROM}$ at $\mathrm{T}_{\mathrm{b}}$ (outcome $_{\text {passiverOM: }}$ : $\mathrm{U}=53, \mathrm{p}=0.483$; outcome active ROM: $\mathrm{U}=64, \mathrm{p}=0.976$ ). However, after three weeks of rehabilitative exercises, the experimental group presented a ROM greater than the control group (outcome $_{\text {passiverOM: }} \mathrm{U}=15, \mathrm{p}=0.001$; outcome activeROM: $_{\text {: }}$ : $\mathrm{U}=22, \mathrm{p}=0.006)$ and showed significant different values respect to pre-treatment ones (outcome passiverOM: $\mathrm{W}=54, \mathrm{p}=0.004 ;$ outcome activerOM: $_{\mathrm{W}} \mathrm{W}=53, \mathrm{p}=0.006$ ). Conversely, the control group presented a significant improvement from $\mathrm{T}_{\mathrm{b}}$ to $\mathrm{T}_{\mathrm{e}}$ only for active ROM (outcome $_{\text {passiveROM: }}: \mathrm{W}=68, \mathrm{p}=0.127 ;$ outcome $_{\text {activerOM: }}$ : $\mathrm{W}=78, \mathrm{p}=0.021$ ).

Detailed results related to single directions are reported in Table 4 and showed in Figs. 5, 6. Their trend

Table 3 Median normalized values (outcome $e_{\text {exercise }}$ ) and IQR of each exercise. For all the exercises, lower values stay for a better performance

\begin{tabular}{|c|c|c|c|c|}
\hline \multirow[t]{2}{*}{ Median values (IQR) } & \multicolumn{2}{|l|}{$T_{b}$} & \multicolumn{2}{|l|}{$\mathrm{T}_{\mathrm{e}}$} \\
\hline & Experimental & Control & Experimental & Control \\
\hline Passive ROM & $0.108(0.202-0.082)$ & $0.161(0.24-0.103)$ & $0.0841(0.0999-0.0501)$ & $0.114(0.241-0.105)$ \\
\hline Active ROM & $0.615(0.631-0.496)$ & $0.619(0.722-0.475)$ & $0.354(0.414-0.171)$ & $0.466(0.521-0.438)$ \\
\hline Isometric force & $0.663(0.809-0.576)$ & $0.836(0.877-0.771)$ & $0.433(0.556-0.309)$ & $0.550(0.759-0.483)$ \\
\hline Target tracking & $0.197(0.288-0.124)$ & $0.207(0.369-0.132)$ & $0.122(0.209-0.089)$ & $0.170(0.279-0.149)$ \\
\hline Joint position matching & $0.298(0.389-0.185)$ & $0.352(0.424-0.288)$ & $0.285(0.456-0.199)$ & $0.318(0.352-0.160)$ \\
\hline
\end{tabular}




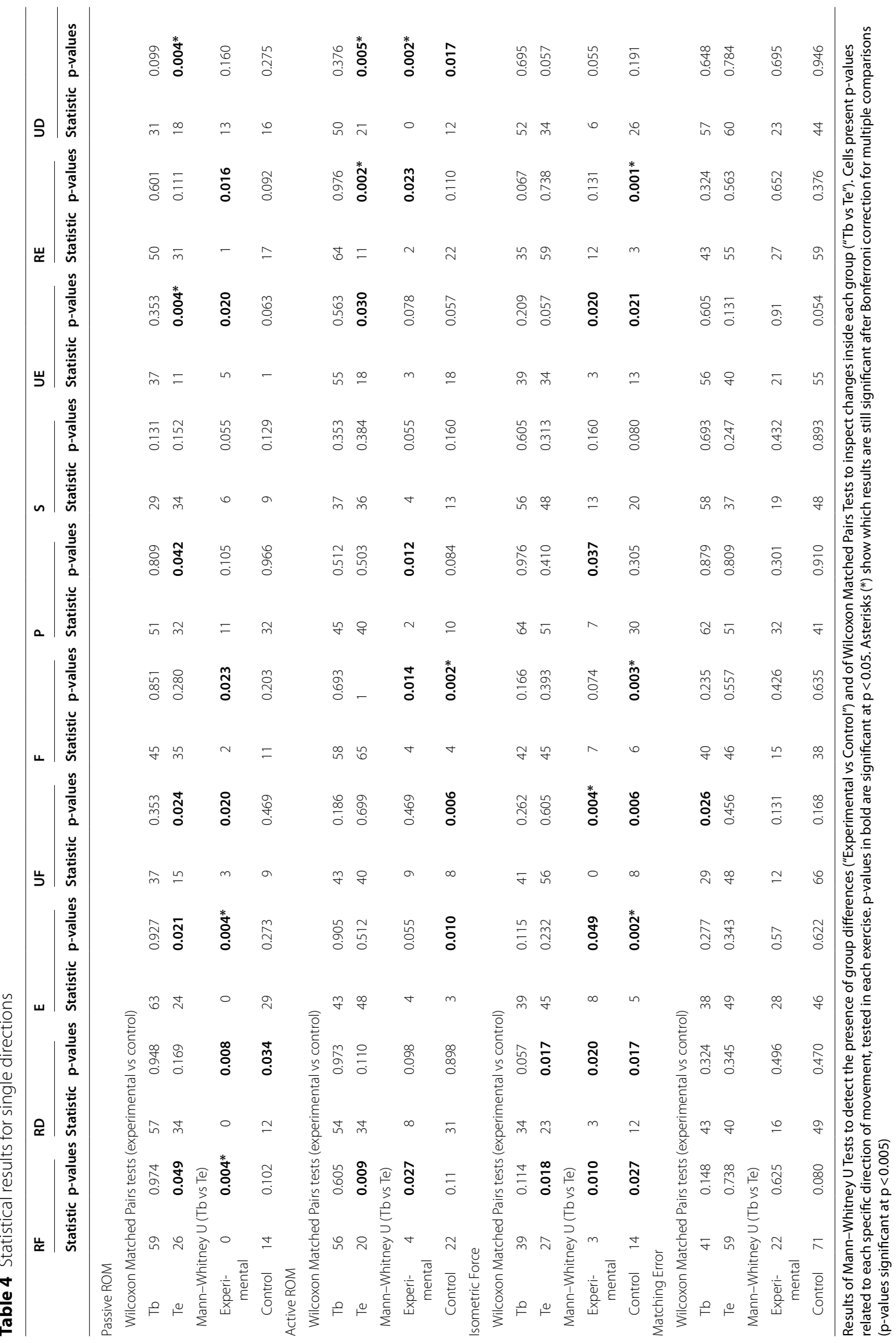



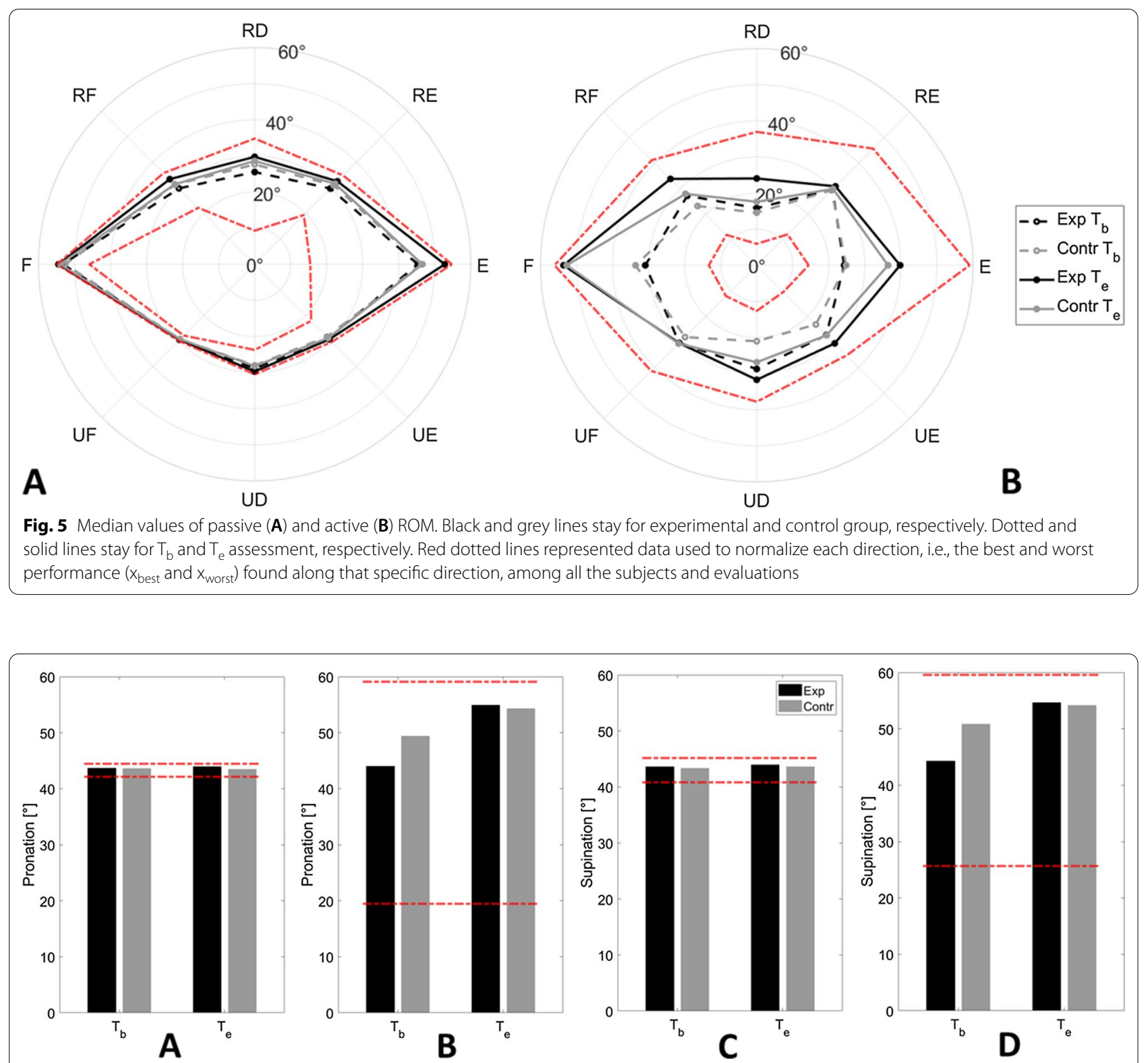

Fig. 6 Median values of passive (A and $\mathbf{C})$ and active (B and $\mathbf{D})$ ROM for pronation ( $\mathbf{A}$ and $\mathbf{B})$ and supination movements $(\mathbf{C}$ and $\mathbf{D})$. Black and grey stay for experimental and control group, respectively. Red dotted lines represented data used to normalize each direction, i.e., the best and worst performance $\left(x_{\text {best }}\right.$ and $\left.x_{\text {worst }}\right)$ found along that specific direction, among all the subjects and evaluations

and significance in both Mann-Whitney $\mathrm{U}$ and Wilcoxon Matched Pairs tests confirmed what has been found from exercise median normalized results (outcome passiveROM $_{1}$

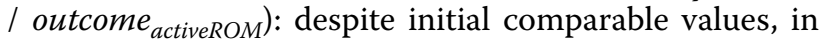
the second evaluation the experimental group showed a larger set of directions presenting significant ROM improvements, particularly in the passive assessment (Table 4).

\section{Isometric force}

Globally, subjects' isometric force (outcome $_{\text {isoforce }}$ ) increased in both groups. Groups presented comparable values of force at $T_{b}(U=38, p=0.101)$, not only considering outcome isoforce, $_{\text {, but also single directions }}$ (Table 4 and Fig. 7). After rehabilitation, both groups improved (experimental: $W=50, p=0.020$; control: $\mathrm{W}=88, \mathrm{p}=0.001)$ : this force growth was similar in the two groups and Mann-Whitney U tests showed 


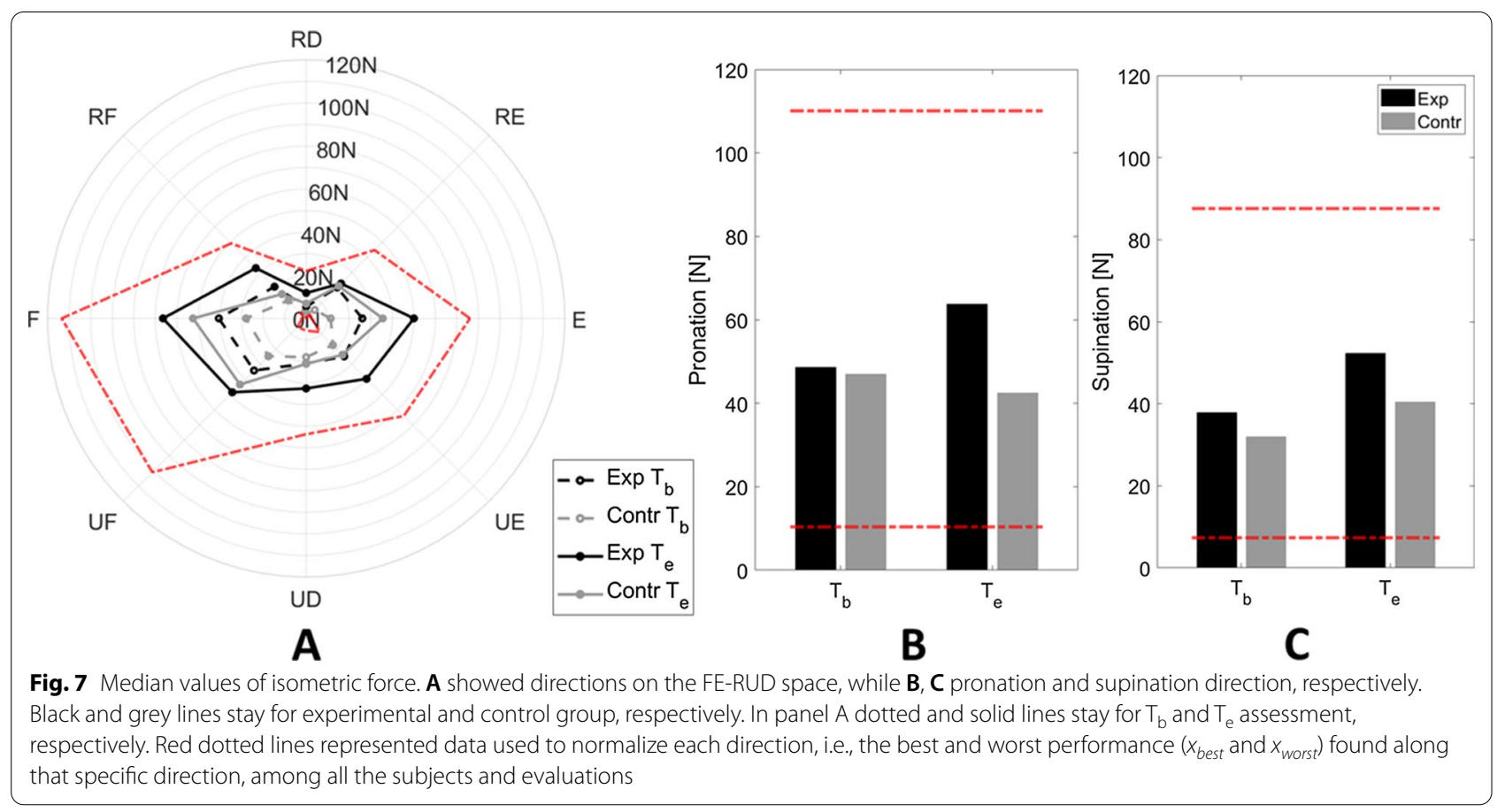

that achieved force values were still comparable at $T_{e}$ $(\mathrm{U}=34, \mathrm{p}=0.057)$ (Table 3).

\section{Target tracking}

Differently from the previous analyzed tasks, target tracking performance was evaluated only through a single outcome measure, i.e., the mean figural error in the two completed laps (see "Subjects and experimental protocol"), without any distinction about movement direction and single DoFs. This indicator metric was processed as described in Eq. 1: normalized median values are shown in Table 3 (outcome $_{\text {tracking }}$ computed with $x_{\text {best }}=0.6^{\circ}, x_{\text {worst }}=5.9^{\circ}$ ), while median figural errors in degrees of each group at each assessment are reported in the following lines. Statistical analysis revealed that target tracking performance was comparable between groups both before $(\mathrm{U}=57, \mathrm{p}=0.648$; experimental: $1.7^{\circ}$, control: $\left.1.7^{\circ}\right)$ and after three weeks of rehabilitation $\left(\mathrm{U}=36, \mathrm{p}=0.077\right.$; experimental: $1.2^{\circ}$, control: $\left.1.5^{\circ}\right)$. Although groups presented comparable final errors, only the experimental group resulted to be improved significantly from $T_{b}$ to $T_{e}$ (experimental: $W=47, p=0.049$; control: $\mathrm{W}=53, \mathrm{p}=0.635$ ).

\section{Joint position matching}

Analogously to what presented for the Target Tracking outcomes, also matching errors were comparable between groups both before $(U=43, p=0.186)$ and after three weeks of rehabilitation $(U=53, p=0.483)$.

As can be guessed by Fig. 8, for the experimental group neither most single directions (Table 4) nor median outcome $_{j p m}$ (Table 3) showed improvements in the perception of wrist position $(\mathrm{W}=22, \mathrm{p}=0.625)$. Conversely, control subjects showed a slight improvement in their performance $(\mathrm{W}=77, \mathrm{p}=0.027)$.

\section{Perceived satisfaction and acceptability}

After the last robotic evaluation, subjects' experienced robotic training and/or assessment was scored with a median value of 10 out of 10 (mean: 9.67, SE: 0.333 ) by the experimental group, and 9.5 (mean: 9.33, SE: 0.333) by the control group. In both groups, no important harm or adverse event was reported.

\section{Discussion}

This randomized controlled trial tested the efficacy of a robot-based rehabilitative protocol to recover wrist functionality after traumatic injuries. The efficacy of robotic devices in the orthopedic field was investigated in a restricted number of studies $[16,17]$, whose main limitation can be found in the absence of a comparison with a traditional treatment. To investigate the efficacy of this novel rehabilitative approach, our protocol involved sessions of assessment pre- and post-rehabilitation, including both robot-aided measurements and evaluations through traditionally used clinical scales. The assessed 


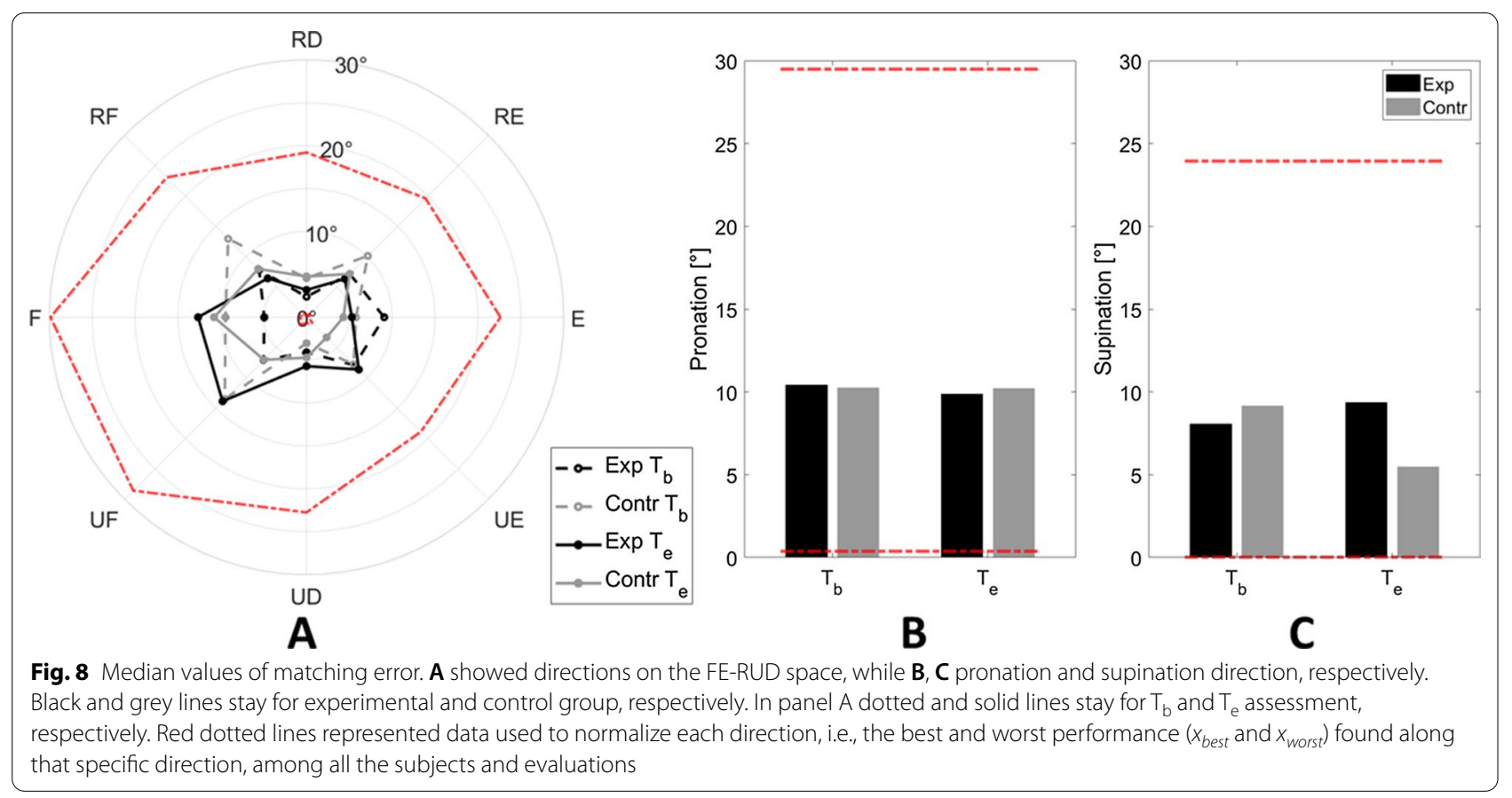

measurements aimed to point out whether robot-based rehabilitation (10 experimental subjects) had an effect, in terms of presence of improvements in functionality, and whether the final performance was comparable to that achieved after a traditional rehabilitative protocol (13 control subjects). The structure of both the robot-based rehabilitative protocol and the traditional one was always chosen by the medical specialist, tailoring the treatment accordingly to the severity and the level of healing achieved by each subject. Robot-based exercises replaced traditional exercises preserving the corresponding goal, such as reducing rigidity [6] or improving stretch, muscle force or dexterity $[7,10,42]$.

Clinical results revealed that the robot-based rehabilitative approach was effective and that its results were comparable to those achieved through traditional exercises. Actually, the function subscale of PRWE pointed out that both robot-aided and traditional rehabilitation led to a recover of wrist functionality and that experimental and control group were comparable both before and after rehabilitation.

The entire set of clinical measures, Jamar Test, JebsenTaylor Test and PRWE, showed that grip force, dexterity, pain, and functionality were comparable between groups, both pre- and post-treatment, stating the comparability between robot-based and traditional approaches. Considering long-term effects, in our study only the control group resulted to have further improved its score in the function subscale of the PRWE at the follow-up assessment. Retaining improvements beyond the period of training is a crucial goal for rehabilitation and evidences of a long-term retention after robotic rehabilitation have already been found in the neuro-rehabilitative field [43-46]. Whether and how retention of functionality is related to the employment of robotic devices for orthopedic rehabilitative treatments should be investigated further.

Robotic assessments showed that both the experimental and the control group significantly improved their performance in robot-based tasks, however, subjects that underwent robot-aided rehabilitation presented a better outcome performance respect to traditionally treated ones. It is crucial to highlight that, since we aimed to avoid speculating on results derived from a more intensive use of the device by the experimental group, tasks used for the robot-aided assessment were designed to be deeply different from those used to treat subjects. Additionally, at each assessment, all subjects had time to familiarize with the device before being tested. Despite this, subjects were also evaluated through clinical scales and their results discussed, removing any possible effect affecting the comparison, related to a more prolonged use of the device.

In details, greater improvements were found in ROM and measures of isometric forces in both groups: subjects' rigidity decreased, allowing wider movements, and muscle force increased in the isometric task. The improvements in ROM and force generation capacity, reported for the experimental group, are in accordance with what was found for the lower limb by Deuthsch 
et al. [17], using a haptic interface for rehabilitation after ankle injuries.

Contrarily, improvements were not so great and clear in the Joint Position Matching and in the Tracking task. It is worth remembering that these tasks were tailored accordingly to subjects' passive ROM. However, the influence of movement amplitude on wrist proprioceptive acuity and tracking accuracy is well known [39, 47]: presence of different ROM among subjects and between assessments, and the subsequent testing with disparate movement amplitudes, could have led to increase the variability of our results, hiding any possible change exclusively related to an improved perception of wrist position. Additionally, another limitation of our study was related to the size of the Lissajous figure considered in the Tracking task: although the size changed accordingly to a percentage of subjects' passive ROM, the tracking task required active movements and, as shown in Fig. 5, at the first evaluation the median active ROM was visibly smaller than the passive one.

Although this study evaluated subjects' performance through indicators widely used in robotic rehabilitation [38, 39], future studies should address the abovepresented issues and, still preserving safe conditions in presence of reduced ROM, find novel tasks and outcome measures of assessment independent from movement amplitude.

Interestingly, even though our sample presented a wide variety and differently treated injuries [1], before rehabilitation groups were comparable in both outcome metrics of each robotic task and clinical measures assessed. Since inclusion criteria did not restricted participants on the basis of the wrist injury or conservative/surgery treatment they got, statistical dispersion of our results was broad: one limitation of this study was related to the high variability of our sample in terms of both sensory and motor performance, that led subjects to be impaired along specific and different directions of movement.

Overall, robotic and clinical results agreed in stating that robot-based rehabilitation was effective and comparable with a traditional protocol. Future studies should address a deeper knowledge about the correlation between clinical and robotic outcomes and whether different robot-based metrics $[22,48]$ could assess other components influencing wrist recovery of functionality in post-traumatic subjects. Although the correlation analysis was not a primary objective of this study, we performed some potentially interesting correlations on our sample of clinical and robotic measures. From preliminary Pearson correlation analyses, we obtained a significant correlation between isometric force (outcome isoforce $_{\text {) }}$ and grip force measured in the Jamar test $(\mathrm{r}=-0.36$, $p=0.013$ ), where the negative correlation results from the processing and computation of outcome isoforce, with lower values indicating higher forces exerted (see "Data Processing"). Additionally, the Robotic Assessment Index resulted significantly correlated with the function subscale of the PRWE $(r=0.326, p=0.033)$, with lower values of both metrics pointing out improved wrist functionality. Even though these analyses showed a relation between novel robotic outcomes and largely employed clinical measures, more interesting and robust results could be obtained in future studies involving larger samples.

Given our results, it is evident that research should particularly focus on developing both somatosensory robot-aided assessments and trainings [19-21], suitable for orthopedic subjects. However, although the proposed robot-aided training employed exercises designed for neuro-rehabilitative purposes $[38,49,50]$, these resulted well-tolerated by post-traumatic subjects. Similarly to what other studies reported using a different robotic device for orthopedic rehabilitation [16], our sample of patients resulted satisfied and well-accepted the device: acceptability was rated with excellent scores, even higher in the experimental group, whose both rehabilitation and assessment were centered on its employment.

The potential of a systematic use of robotic devices in orthopedics is twofold: besides increasing accuracy and repeatability in the assessment of functionality, robotbased rehabilitation could be maximally exploited tailoring rehabilitative protocols real-time to target to subject's specific functional deficits and promote his/her voluntary participation [51], minimizing time duration and therapists' effort during rehabilitation.

\section{Conclusions}

This work aimed to test a robot-based rehabilitative approach on orthopedic subjects. Our results showed that the robot-aided protocol of treatment was effective and comparable to the traditional one. Despite our sample presented a wide variety of wrist injuries, subjects' wrist functionality was comparable before the treatment considering both robotic evaluations and assessed clinical measures and scales. After the three-week long rehabilitation, clinical results showed that groups did not differ in terms of functionality, pain, grip force and dexterity. The robotic assessment showed that the experimental group presented greater improvements than the control group, particularly in terms of reduced tissue rigidity and increased muscle force. This work can be considered as a starting point for introducing the use of robotic devices in the orthopedic field, where a systematic use of these devices could assist therapists' work and 
increase accuracy in tailoring treatments to target specific injury-related issues.

\section{Acknowledgements}

The authors wish to thank all the subjects who took part to the clinical trial and the institutes that supported this research.

\section{Authors' contributions}

JZ, LM, ET, GS and PC designed the study. JZ implemented the software used in the work. CG, SG, FP and CS collected data and supervised the treatment. GAA analyzed and interpreted the data, performed statistical analysis, and wrote the manuscript. JZ and ET supervised the study. JZ helped with data interpretation and manuscript drafting. All authors approved the final version of the manuscript. All authors read and approved the final manuscript.

\section{Funding}

This work has been supported by the National Institute for Insurance against Accidents (INAIL) and the Robotics, Brain and Cognitive Sciences Unit of the Istituto Italiano di Tecnologia.

\section{Availability of data and materials}

The datasets used and/or analyzed during the current study are available from the corresponding author on reasonable request.

\section{Declarations}

\section{Ethics approval and consent to participate}

Written informed consent was obtained from the subjects for publication of this study and any accompanying images. The study was approved by the Ethics Committee of the regional health authority (protocol number 76, code CRMINAILO3).

\section{Consent for publication}

Consent to publish was obtained from participants.

\section{Competing interests}

The authors declare that they have no competing interests.

\begin{abstract}
Author details
${ }_{1}^{1}$ Robotics, Brain and Cognitive Sciences (RBCS) Unit, Istituto Italiano di Tecnologia, Genoa, Italy. ${ }^{2}$ Department of Informatics, Bioengineering, Robotics and Systems Engineering (DIBRIS), University of Genoa, Genoa, Italy. ${ }^{3}$ National Institute for Insurance against Accidents at Work (INAIL), Motor Rehabilitation Center, Volterra, Italy. ${ }^{4}$ Institut für Technische Informatik (ZITI), Heidelberg University, Heidelberg, Germany.
\end{abstract}

Received: 4 March 2021 Accepted: 23 August 2021

Published online: 31 August 2021

\section{References}

1. Jaworski CA, Krause M, Brown J. Rehabilitation of the wrist and hand following sports injury. Clin Sports Med. 2010;29(1):61-80. https://doi.org/ 10.1016/j.csm.2009.09.007.

2. Barrack RL, Skinner HB, Buckley SL. Proprioception in the anterior cruciate deficient knee. Am J Sports Med. 1989;17(1):1-6. https://doi.org/10.1177/ 036354658901700101.

3. Karagiannopoulos C, Sitler M, Michlovitz S, Tierney R. A descriptive study on wrist and hand sensori-motor impairment and function following distal radius fracture intervention. J Hand Ther. 2013;26(3):204-15. https:// doi.org/10.1016/j.jht.2013.03.004.

4. Riemann BL, Lephart SM. The sensorimotor system, Part II: the role of proprioception in motor control and functional joint stability. J Athl Train. 2002;37(1):80-4. https://doi.org/10.1016/j.jconhyd.2010.08.009.

5. Pappou IP, Basel J, Deal DN. Scapholunate ligament injuries: a review of current concepts. Hand. 2013;8(2):146-56. https://doi.org/10.1007/ s11552-013-9499-4
6. Albanese GA, Marini F, Taglione E, et al. Assessment of human wrist rigidity and pain in post-traumatic patients. IEEE Int Conf Rehabil Robot. 2019 https://doi.org/10.1109/ICORR.2019.8779508.

7. Bruder AM, Taylor NF, Dodd KJ, Shields N. Physiotherapy intervention practice patterns used in rehabilitation after distal radial fracture. Physiother (United Kingdom). 2013;99(3):233-40. https://doi.org/10.1016/j. physio.2012.09.003.

8. Krischak GD, Krasteva A, Schneider F, Gulkin D, Gebhard F, Kramer M. Physiotherapy after volar plating of wrist fractures is effective using a home exercise program. Arch Phys Med Rehabil. 2009;90(4):537-44. https://doi.org/10.1016/j.apmr.2008.09.575.

9. Bruder A, Taylor NF, Dodd KJ, Shields N. Exercise reduces impairment and improves activity in people after some upper limb fractures: a systematic review. J Physiother. 2011;57(2):71-82. https://doi.org/10.1016/S18369553(11)70017-0

10. Handoll HH, Madhok R, Howe TE. Rehabilitation for distal radial fractures in adults. Cochrane Database Syst Rev. 2006. https://doi.org/10.1002/ 14651858.CD003324.pub2.

11. Saedén B, Törnkvist H, Ponzer S, Höglund M. Fracture of the carpal scaphoid. A prospective, randomised 12-year follow-up comparing operative and conservative treatment. J Bone Joint Surg Br. 2001;83(2):230-4. https://doi.org/10.1302/0301-620x.83b2.11197.

12. Glasgow C, Tooth LR, Fleming J. Mobilizing the stiff hand: Combining theory and evidence to improve clinical outcomes. J Hand Ther. 2010;23(4):392-401. https://doi.org/10.1016/j.jht.2010.05.005.

13. Volpe BT, Krebs HI, Hogan N, Edelstein L, Diels C, Aisen M. A novel approach to stroke rehabilitation. Neurology. 2000;54(10):1938-44. https://doi.org/10.1212/WNL.54.10.1938.

14. Krebs HI, Mernoff S, Fasoli SE, Hughes R, Stein J, Hogan N. A comparison of functional and impairment-based robotic training in severe to moderate chronic stroke: a pilot study. NeuroRehabilitation. 2008;23:81-7. https://doi.org/10.3233/NRE-2008-23108.

15. Ferraro M, Palazzolo JJ, Krol J, Krebs HI, Hogan N, Volpe BT. Robot-aided sensorimotor arm training improves outcome in patients with chronic stroke. Neurology. 2003;61(11):1604-7. https://doi.org/10.1212/01.WNL. 0000095963.00970 .68$.

16. Padilla-Castañeda MA, Sotgiu E, Barsotti M, et al. An orthopaedic roboticassisted rehabilitation method of the forearm in virtual reality physiotherapy. J Healthc Eng. 2018. https://doi.org/10.1155/2018/7438609.

17. Deutsch JE, Latonio J, Burdea GC, Boian R. Rehabilitation of musculoskeletal injuries using the Rutgers ankle haptic interface: three case reports. In: EuroHaptics Conference. Birmingham, UK; 2001. pp. 11-6.

18. Marchal-Crespo L, Reinkensmeyer DJ. Review of control strategies for robotic movement training after neurologic injury. J Neuroeng Rehabil. 2009;6(1):20. https://doi.org/10.1186/1743-0003-6-20.

19. Cuppone AV, Squeri V, Semprini M, Masia L, Konczak J. Robot-assisted proprioceptive training with added vibro-tactile feedback enhances somatosensory and motor performance. PLOS ONE. 2016;11(10):1-18. https://doi.org/10.1371/journal.pone.0164511.

20. De Santis D, Zenzeri J, Casadio M, et al. Robot-assisted training of the kinesthetic sense: enhancing proprioception after stroke. Front Hum Neurosci. 2015;8(January):1-12. https://doi.org/10.3389/fnhum.2014. 01037.

21. Casadio M, Morasso P, Sanguineti V, Giannoni P. Minimally assistive robot training for proprioception enhancement. Exp Brain Res. 2009;194(2):219-31. https://doi.org/10.1007/s00221-008-1680-6.

22. Falzarano V, Petrella G, Marini F, et al. Preliminary evaluation of a robotic measurement system for the assessment of wrist joint spasticity. Proc IEEE RAS EMBS Int Conf Biomed Robot Biomechatronics. 2020. https:// doi.org/10.1109/BioRob49111.2020.9224343.

23. Mugnosso M, Zenzeri J, Hughes CML, Marini F. Coupling robot-aided assessment and surface electromyography (sEMG) to Evaluate the effect of muscle fatigue on wrist position sense in the flexion-extension plane. Front Hum Neurosci. 2019;13(November):1-12. https://doi.org/10.3389/ fnhum.2019.00396

24. Jack D, Boian R, Merians AS, et al. Virtual reality-enhanced stroke rehabilitation. IEEE Trans Neural Syst Rehabil Eng. 2001;9(3):308-18. https://doi. org/10.1109/7333.948460.

25. Goršič M, Cikajlo I, Novak D. Competitive and cooperative arm rehabilitation games played by a patient and unimpaired person: effects on 
motivation and exercise intensity. J Neuroeng Rehabil. 2017;14(1):1-18. https://doi.org/10.1186/s12984-017-0231-4.

26. Fluet GG, Merians AS, Qiu Q, Davidow A, Adamovich SV. Comparing integrated training of the hand and arm with isolated training of the same effectors in persons with stroke using haptically rendered virtual environments, a randomized clinical trial. J Neuroeng Rehabil. 2014;11(1):1-11. https://doi.org/10.1186/1743-0003-11-126.

27. Masia L, Casadio M, Sandini G, Morasso P. Eye-hand coordination during dynamic visuomotor rotations. PLoS ONE. 2009. https://doi.org/10.1371/ journal.pone.0007004.

28. Iandolo R, Marini F, Semprini M, et al. Perspectives and challenges in robotic neurorehabilitation. Appl Sci. 2019. https://doi.org/10.3390/ app9153183.

29. Falzarano V, Marini F, Morasso P, Zenzeri J. Devices and protocols for upper limb robot-assisted rehabilitation of children with neuromotor disorders. Appl Sci. 2019;9(13):1-22. https://doi.org/10.3390/app9132689.

30. Cuppone A, Squeri V, Semprini M, Konczak J. Robot-assisted training to improve proprioception does benefit from added vibro-tactile feedback. Proc Annu Int Conf IEEE Eng Med Biol Soc EMBS. 2015. https://doi.org/10. 1109/EMBC.2015.7318349.

31. Albanese GA, Holmes MWR, Marini F, Morasso P, Zenzeri J. Wrist position sense in two dimensions: between-hand symmetry and anisotropic accuracy across the space. Front Hum Neurosci. 2021;15:202. https://doi. org/10.3389/FNHUM.2021.662768.

32. Bechtol CO. Grip test; the use of a dynamometer with adjustable handle spacings. J Bone Joint Surg Am. 1954;36-A(4):820-4.

33. Jebsen RH, Taylor N, Trieschmann RB, Trotter MJ, Howard LA. An objective and standardized test of hand function. Arch Phys Med Rehabil. 1969;50(6):311-9.

34. Davis Sears E, Chung KC. Validity and responsiveness of the jebsen-taylor hand function test. J Hand Surg Am. 2010;35(1):30-7. https://doi.org/10. 1016/j.jhsa.2009.09.008.

35. MacDermid JC, Richards RS, Donner A, Bellamy N, Roth JH. Responsiveness of the SF-36, DASH, PRWE, and physcial impairment measurements in evaluating recovery after a distal radius fracture. J Hand Surg Am. 2000;25:330-40.

36. MacDermid JC, Tottenham V. Responsiveness of the disability of the arm, shoulder, and hand (DASH) and patient-rated wrist/hand evaluation (PRWHE) in evaluating change after hand therapy. J Hand Ther. 2004:17(1):18-23. https://doi.org/10.1197/j.jht.2003.10.003.

37. MacDermid JC, Richards RS, Donner A, Bellamy N, Roth JH. Responsiveness of the short form-36, Disability of the Arm, Shoulder, and Hand questionnaire, patient-rated wrist evaluation, and physical impairment measurements in evaluating recovery after a distal radius fracture. J Hand Surg Am. 2000;25(2):330-40. https://doi.org/10.1053/jhsu.2000.jhsu2 $5 \mathrm{a} 0330$.

38. Squeri V, Masia L, Casadio M, Morasso P, Vergaro E. Force-field compensation in a manual tracking task. PLoS ONE. 2010. https://doi.org/10.1371/ journal.pone.0011189.

39. Marini F, Squeri V, Morasso P, Konczak J, Masia L. Robot-aided mapping of wrist proprioceptive acuity across a 3D workspace. PLoS ONE. 2016;1 1(8):1-12. https://doi.org/10.1371/journal.pone.0161155.

40. van Berckel MMG, Bosma NH, Hageman MGJS, Ring D, Vranceanu AM. The correlation between a numerical rating scale of patient satisfaction with current management of an upper extremity disorder and a general measure of satisfaction with the medical visit. Hand. 2017;12(2):202-6. https://doi.org/10.1177/1558944716662019.

41. Hjermstad MJ, Fayers PM, Haugen DF, et al. Studies comparing numerical rating scales, verbal rating scales, and visual analogue scales for assessment of pain intensity in adults: a systematic literature review. J Pain Symptom Manage. 2011;41(6):1073-93. https://doi.org/10.1016/j.jpain symman.2010.08.016.

42. Ikpeze TC, Smith HC, Lee DJ, Elfar JC. Distal radius fracture outcomes and rehabilitation. Geriatric Orthopaedic Surg Rehabil. 2016;7(4):202-5. https://doi.org/10.1177/2151458516669202.

43. Casadio M, Sanguineti V. Learning, retention, and slacking: a model of the dynamics of recovery in robot therapy. IEEE Trans Neural Syst Rehabil Eng. 2012;20(3):286-96. https://doi.org/10.1109/TNSRE.2012.2190827.

44. Fasoli SE, Krebs HI, Stein J, Frontera WR, Hughes R, Hogan N. Robotic therapy for chronic motor impairments after stroke: follow-up results $11 \mathrm{An}$ organization, with which 1 or more of the authors is associated, has received or will receive financial benefits from a commercial party having a direct financial interest i. Arch Phys Med Rehabil. 2004;85(7):1 106-11. https://doi.org/10.1016/j.apmr.2003.11.028.

45. Masiero S, Celia A, Rosati G, Armani M. Robotic-assisted rehabilitation of the upper limb after acute stroke. Arch Phys Med Rehabil. 2007;88(2):1429. https://doi.org/10.1016/j.apmr.2006.10.032.

46. Lum PS, Burgar CG, Shor PC, Majmundar M, Van der Loos M. Robotassisted movement training compared with conventional therapy techniques for the rehabilitation of upper-limb motor function after stroke. Arch Phys Med Rehabil. 2002;83(7):952-9. https://doi.org/10.1053/apmr. 2001.33101.

47. Marini F, Cappello L, Squeri V, et al. Online modulation of assistance in robot aided wrist rehabilitation a pilot study on a subject affected by dystonia. IEEE Haptics Symp HAPTICS. 2014. https://doi.org/10.1109/ HAPTICS.2014.6775448.

48. Mugnosso M, Marini F, Holmes M, Morasso P, Zenzeri J. Muscle fatigue assessment during robot-mediated movements. J Neuroeng Rehabil. 2018;15(1):119. https://doi.org/10.1186/s12984-018-0463-y.

49. Masia L, Casadio M, Giannoni P, Sandini G, Morasso P. Performance adaptive training control strategy for recovering wrist movements in stroke patients: a preliminary, feasibility study. J Neuroeng Rehabil. 2009;11:111. https://doi.org/10.1186/1743-0003-6-44.

50. Squeri V, Masia L, Giannoni P, Sandini G, Morasso P. Wrist rehabilitation in chronic stroke patients by means of adaptive, progressive robot-aided therapy. IEEE Trans Neural Syst Rehabil Eng. 2014;22(2):312-25. https:// doi.org/10.1109/TNSRE.2013.2250521.

51. Vergaro E, Casadio M, Squeri V, Giannoni P, Morasso P, Sanguineti V. Self-adaptive robot training of stroke survivors for continuous tracking movements. J Neuroeng Rehabil. 2010;7(1):1-12. https://doi.org/10.1186/ 1743-0003-7-13.

\section{Publisher's Note}

Springer Nature remains neutral with regard to jurisdictional claims in published maps and institutional affiliations.

Ready to submit your research? Choose BMC and benefit from

- fast, convenient online submission

- thorough peer review by experienced researchers in your field

- rapid publication on acceptance

- support for research data, including large and complex data types

- gold Open Access which fosters wider collaboration and increased citations

- maximum visibility for your research: over 100M website views per year

At BMC, research is always in progress.

Learn more biomedcentral.com/submissions 